\title{
ON THE COMPACTIFICATION OF STRONGLY PSEUDOCONVEX SURFACES. II
}

\author{
VO VAN TAN ${ }^{1}$
}

\begin{abstract}
A complete answer to the following question is given: When is an algebraic surface $M$ a compactification of some strongly pseudoconvex surface? In particular, we show this will not be the case if $M$ is either $\mathbf{P}_{2}$, a quadric, an abelian surface, or a hyperelliptic surface. On the other hand, by constructing specific examples, we show that this will be the case for all other algebraic surfaces. Furthermore, we prove that any compactifiable strongly pseudoconvex surface is quasi-projective.
\end{abstract}

In [10] we showed that a compactification of a strongly pseudoconvex surface is either an algebraic surface or a (highly) nonalgebraic surface, namely some Inoue surface. Moreover, we exhibited an example showing that indeed those two alternatives did occur (even simultaneously). Here we would like to further pursue our investigation in that direction. In the first part of this paper, we explore what algebraic surfaces really do (or do not) occur as compactifications of some strongly pseudoconvex surfaces. In the second part, we study the algebraic structure of compactifiable strongly pseudoconvex surfaces.

Throughout we shall freely use the definitions and notations introduced in [10]. In particular, all $\mathbf{C}$-analytic surfaces (compact or noncompact) are assumed to be minimal.

\section{The algebraic compactification.}

Definition 1 [4]. Let $X$ be a $\mathbf{C}$-analytic manifold. Then $X$ is said to be strongly pseudoconvex if

(i) $X$ is holomorphically convex,

(ii) there exists a compact analytic subvariety $E \subset X$ with $\operatorname{dim}_{x} E>0$ for any $x \in E$ such that $T \subset E$ for any positive-dimensional and irreducible compact analytic subvariety $T$ in $X$.

REMARK. One can check that this definition for strongly pseudoconvex manifolds is equivalent to the one given in [10] (see e.g. [4]). Furthermore, $E$ is called the

Received by the editors March 15, 1983.

1980 Mathematics Subject Classification. Primary 14J10, 32J05, 32J15; Secondary 32E05, 32F10.

Key words and phrases. Classification of algebraic surfaces, canonical dimension, compactifiable strongly pseudoconvex surfaces, quasi-projective structure, affine $C$-bundle over elliptic curve.

'Partially supported by a Faculty Development Grant at the University of Massachusetts, Boston (1981-1982). The author would like to thank the referee for suggestions which simplified the original version. 
exceptional set of $X$. Note that, unless the contrary is explicitly stated, we always assume, as in [10], that $E \neq \varnothing$, i.e. $X$ is not Stein.

Proposition 1. Let $M$ be a compact homogeneous $\mathbf{C}$-analytic manifold. Then $M$ cannot be the compactification of any strongly pseudoconvex manifold $X$.

Proof. Let $\operatorname{Aut}(M)$ be the group all holomorphic automorphisms of $M$ and let $\operatorname{Aut}(M)^{\circ}$ be the connected component of $\operatorname{Aut}(M)$ containing the identity element.

Claim. $M$ is not the compactification of any strongly pseudoconvex manifold $X$.

In fact, if it were, let $\Gamma$ be the compact analytic subvariety in $M$ such that $M \backslash \Gamma \simeq X$; since $X$ is strongly pseudoconvex, $\Gamma$ is of pure codimension one. Now let $E$ be the exceptional set of $X$. Certainly $E \cap \Gamma=\varnothing$. Without loss of generality, one can assume that $E$ is irreducible. Since $M$ is homogeneous, a main result in [9] tells us there exists a $t \in \operatorname{Aut}(M)^{\circ}$ such that $t(E)=: T \nsubseteq E$. Since $\operatorname{dim} T=\operatorname{dim} E>0$, this contradicts the maximality condition (ii) of Definition 1. Hence our claim is proved. Q.E.D.

Our main focus here is the following

Problem 1. What are the possible algebraic compactifications $M$ of strongly pseudoconvex surfaces $X$ ?

Before tackling this problem, a brief review of the classification of compact algebraic surfaces is in order (see [8a-c]).

Definition 2. [5]. Let $M$ be a compact $\mathbf{C}$-analytic manifold. Then the canonical dimension of $M$, denoted by $\kappa(M)$, is defined to be the transcendence degree over $\mathbf{C}$ of the quotient field of the graded ring $\bigoplus_{n=0}^{\infty} H^{\circ}(M, O(n K))$ minus one, where $K$ is the canonical bundle on $M$.

For compact algebraic surfaces $M$, it is known that $\kappa(M)=-1,0,1$ or 2 . According to their canonical dimensions, compact algebraic surfaces are completely classified as follows.

THEOREM 2 [8b] (SEE ALSO [5]). Let $M$ be a compact algebraic surface. Then $M$ must be one of the following types:

(a) If $\kappa(M)=-1$ :

(i) $a \mathbf{P}_{2}$,

(ii) a rational surface, or

(iii) a nonrational ruled surface;

(b) If $\kappa(M)=0$ :

(iv) an abelian surface, i.e. algebraic surface which is biholomorphic to a complex 2-dimensional torus;

(v) a hyperelliptic surface, i.e. algebraic surface fibred over $\mathbf{P}_{1}$ by a pencil of elliptic curves;

(vi) a K-3 surface, i.e. algebraic surface which is simply connected and whose canonical bundle is trivial; or

(vii) an Enriques surface, i.e. a quotient of some $K-3$ surface by a fixed point free involution;

(c) If $\kappa(M)=1$; 
(viii) an elliptic surface, i.e. an algebraic surface with a morphism $\pi$ from $M$ to a curve $\Theta$ such that almost all fibres of $\pi$ are nonsingular elliptic curves;

(d) If $\kappa(M)=2$;

(ix) a surface of general type, i.e. a surface $M$ such that, for some $n>0,|n K|$ determines a birational morphism of $M$ onto its image in $\mathbf{P}_{N}$.

We are now in a position to provide a complete answer to Problem 1 within the framework of Theorem 2.

THEOREM A. Let $M$ be an algebraic compactification of some strongly pseudoconvex surface $X$. Then $M$ can be neither a $\mathbf{P}_{2}$, nor an abelian surface, nor a hyperelliptic surface.

Proof. In view of Proposition $1, M$ cannot be $\mathbf{P}_{2}$, nor $\mathbf{P}_{1} \times \mathbf{P}_{1}$, nor an abelian surface. On the other hand, it is known [5] that any hyperelliptic surface $M$ admits an unbranched finite covering $\tilde{M}$ which is an abelian surface. But one can easily show that $M$ is a compactification of some strongly pseudoconvex surface if and only if $\tilde{M}$ is the one. Again in view of Proposition 1, our proof is concluded. Q.E.D.

We would now like to exhibit examples showing that the remaining 6 possibilities for $M$ in Theorem 2 indeed occur.

Example B. (a) Let $X$ be a line bundle on $\mathbf{P}_{1}$ with $c_{1}(X)=-n, n>1$. It is known that $X$ is a strongly pseudoconvex surface [4]. By projectivizing the fibres of $X$, one obtains a $\mathbf{P}_{1}$-bundle over $\mathbf{P}_{1}$, say $M$ which is certainly a rational compactification of $X$.

(b) In [10] we exhibited a nonrational ruled surface $M$ which is the compactification of some strongly pseudoconvex surface $X$ (see also $\$ 2$ below).

(c) Let $\Sigma$ be the Kummer surface in $P_{3}$ (see e.g. [5]) having 16 double points, say $\sigma$. Let $H$ be a hyperplane divisor on $\Sigma$ such that $H \cap \sigma=\varnothing$. Let $M$ be the minimal desingularization of $\Sigma$ and let $\pi: M \rightarrow \Sigma$ be the resulting map inducing a biholomorphism $M \backslash E \simeq \Sigma \backslash \sigma$ where $E:=\pi^{-1}(\sigma)$. Let $\Gamma:=\pi^{-1}(H)$. One can check that $M$ is a $K-3$ surface [5] which is a compactification of the strongly pseudoconvex surface $X:=M \backslash \Gamma$.

(d) Let us specify 9 points, say $\left\{x_{i}\right\}, 1 \leqslant i \leqslant 9$, in $\mathbf{P}_{2}$ forming the base locus of a pencil of cubics $\left\{C_{k}\right\}$. Let $H$ be a hyperplane in $\mathbf{P}_{2}$ such that $x_{i} \notin H$ for any $i$, and let us specify 2 cubics, say $C_{1}$ and $C_{2}$ which intersect transversally. Let $\theta: \tilde{\mathbf{P}}_{2} \rightarrow \mathbf{P}_{2}$ be the blow-up of $\mathbf{P}_{2}$ at those 9 points, and let $\tilde{C}_{1}$ (resp. $\tilde{C}_{2}$ ) be the proper transform of $C_{1}$ (resp. $C_{2}$ ) by $\theta$. Now let us perform a logarithmic transformation of order 2 at $\tilde{C}_{1}$ (resp. $\tilde{C}_{2}$ ) (see e.g. [5, 8a, b]). Let $\lambda: M \rightarrow \tilde{\mathbf{P}}_{2}$ be the resulting map and let $\pi:=\theta \circ \lambda$ : $M \rightarrow \mathbf{P}_{2}$. Let $\Gamma:=\pi^{-1}(H)$. Then one can check that $M$ is an Enriques surface [5] which is a compactification of the strongly pseudoconvex surface $X:=M \backslash \Gamma$.

(e) Let $M$ be an elliptic surface such that one of its singular, reducible fibres contains an irreducible component $E$ which is a rational nonsingular curve with $E^{2}=-2$ (see e.g. [8a]). In view of [1], by contracting $E$ to a point, one obtains a compact surface $S$ with only one isolated singular point. Let $\pi: M \rightarrow S$ be the 
blow-down map inducing a biholomorphism $M \backslash E \simeq S \backslash\{*\}$. Since $E$ is nonsingular and rational, and since $M$ is nonsingular, a main result in [1] tells us that $S$ is projective algebraic. Now let $H$ be a hyperplane divisor in $S$ not meeting the singular point. Then one can check that $M$ is the compactification of the strongly pseudoconvex surface $X:=M \backslash \Gamma$, where $\Gamma:=\pi^{-1}(H)$.

(f) Let $M$ be a given surface of general type. Then for any integer $n>0$, let $\pi:=\Phi_{n K}: M \rightarrow \mathbf{P}_{N}$ be the rational map determined by the pluricanonical system $|n K|$ with $N:=\operatorname{dim}|n K|$. Since $\kappa(M)=2$, a main result in [2] (see also [8c]) tells us that for $n \geqslant 5$;

(i) $S:=\pi(M)$ is an algebraic surface with only isolated singular points, say $\sigma$;

(ii) $\pi$ is a holomorphic birational map, inducing a biholomorphism $M \backslash E \simeq S \backslash \sigma$, where $E:=\pi^{-1}(\sigma)$.

So let $H$ be a hyperplane divisor in $S$ such that $H \cap \sigma=\varnothing$ and let $\Gamma:=\pi^{-1}(H)$. Then one can check that $M$ is actually the compactification of the strongly pseudoconvex surface $X:=M \backslash \Gamma$.

2. The quasi-projective structure. In [10] we exhibited a strongly pseudoconvex surface $X$ which admits two distinct compactifications: one, $M^{\prime}$, highly nonalgebraic, and the other, $M$, and (algebraic) ruled surface. Hence, one would like to raise the following

Problem 2. Is any compactifiable strongly pseudoconvex surface algebraic?

Our main purpose here it to provide an affirmative answer to Problem 2; in particular, this shows that the example exhibited in [10] is by no means an accident.

Recently, Enoki [3] constructed a family of compact $\mathbf{C}$-analytic surfaces, denoted by $S_{n, \alpha, t}$ with $n>0,0<|\alpha|<1$ and $t \in \mathbf{C}^{n}$, having the following properties:

(i) $b_{1}\left(S_{n, \alpha, t}\right)=1$;

(ii) $b_{2}\left(S_{n, \alpha, t}\right)=n$;

(iii) there exists a divisor $\Gamma$ on $S_{n, \alpha, t}$ such that $\Gamma^{2}=0$.

Remark. Some compact $\mathbf{C}$-analytic surface of this type was first constructed by Inoue [7], namely the surface $S_{1, \alpha, 0}$, which has been used by us as a prototype in [10].

Furthermore, the following crucial result has been established.

Theorem 3 [3]. Let $M$ be a compact $\mathbf{C}$-analytic surface. Let us assume

(i) $b_{1}(M)=1$;

(ii) $b_{2}(M)=n>0$;

(iii) there exists a divisor $D \neq 0$ on $M$ such that $D^{2}=0$.

Then $M$ is biholomorphic to some $S_{n, \alpha, t}$ and $D=t \Gamma$ for some integer $t \neq 0$.

We are now in a position to prove the main result of this section.

THEOREM C. Let $X$ be a compactifiable strongly pseudoconvex surface. Then $X$ is quasi-projective.

Proof. Let $M$ be a compactification of $X$ and $\Gamma$ a compact $\mathbf{C}$-analytic curve in $M$ such that $X \simeq M \backslash \Gamma$. A main result in [10] tells us that $M$ is either

(a) algebraic, or

(b) $b_{2}(M) \geqslant b_{1}(M)=1$ and $a(M)=0$. 
Now if $M$ is of type (a) then certainly $X$ is quasi-projective. So let us assume $M$ is of type (b). Since $X$ is strongly pseudoconvex, hence holomorphically convex, and since $a(M)=0$, an argument in [6] (see also [8b]) tells us there exists a divisor $D$ supporting on $\Gamma$ such that $D^{2}=0$. Theorem 3 then tells us $M \simeq S_{n, \alpha, t}$ for some $n, \alpha$ and $t$. Now by construction (see [3,7]) $X \simeq M \backslash \Gamma \simeq S_{n, \alpha, t} \backslash \Gamma$ is an affine C-bundle over some elliptic curve $E$. Hence $X$ is quasi-projective. Q.E.D.

Definition 3. A strongly pseudoconvex manifold $X$ is called Stein if

$$
\operatorname{dim} E \leqslant 0 \text {. }
$$

Corollary D. ${ }^{2}$ Any compactifiable Stein surface $X$ is algebraic.

Proof. Let $M$ be a compactification of $X$ and $\Gamma$ a compact analytic curve in $M$ such that $X \simeq M \backslash \Gamma$. A main result in [6] tells us $M$ is either

(a) algebraic, or

(b) $b_{1}(M)=1$ and $a(M)=0$.

Now if $M$ is of type (a), certainly $X$ is algebraic. So let us assume $M$ is of type (b). Now the same argument as in the proof of Theorem $\mathrm{C}$, implies the existence of a divisor $D$ supporting on $\Gamma$ such that $D^{2}=0$.

Claim. $b_{2}(M)=0$.

In fact, if $b_{2}(M)>0$, then Theorem 3 tells us $M \simeq X \backslash \Gamma$ is an affine C-bundle over some elliptic curve $E$; but this will contradict $(\dagger)$. Therefore $b_{2}(M)=0$, which implies, in view of a result of Kodaira [8b], that $M$ is a nonelliptic Hopf surface. Since $X$ is Stein, $M$ must contain exactly one irreducible elliptic curve [8b]. However, for such a Hopf surface $M$, a biholomorphic map $\pi: M \rightarrow \mathbf{C}^{2} \backslash\left\{z_{1} z_{2}=0\right\}$ was explicitly constructed in [6]. Consequently, $X$ is algebraic. Q.E.D.

To round off this discussion, we would like to mention two related problems.

Problem 3. Construct a strongly pseudoconvex surface $X$ admitting two algebraic compactifications $M_{1}$ and $M_{2}$ which are not birationally equivalent?

In the special case where $X$ is Stein, an affirmative answer to Problem 3 is well known.

Problem 4. Let $M_{1}$ and $M_{2}$ be two nonalgebraic compactifications of some strongly pseudoconvex surface $X$. Are $M_{1}$ and $M_{2}$ bimeromorphically equivalent?

ACKNOWLEDGEMENT. During the final stage this paper was also supported by an NSF grant.

\section{REFERENCES}

1. M. Artin, Some numerical criteria for contractibility of curves on algebraic surfaces, Amer. J. Math. 84 (1962), 485-496.

2. E. Bombieri, Canonical models of surfaces of general type, Inst. Hautes Études Sci. Publ. Math. 42 (1973), 441-445.

3. I. Enoki, Surfaces of class VII with curves, Tôhoku Math. J. (2) 33 (1981), 453-492.

4. H. Grauert, Über Modifikationen und exzeptionelle analytische Mengen, Math. Ann. 146 (1962), 331-368.

5. P. Griffiths and J. Harris, Principles of algebraic geometry, Wiley, New York, 1978.

6. A. Howard, On the compactification of Stein surfaces, Math. Ann. 176 (1968), 221-224.

\footnotetext{
${ }^{2}$ This answers affirmatively a question posed to the author by A. Howard in 1976.
} 
7. M. Inoue, New surfaces with no meromorphic functions, Proc. Internat. Congr. Math. (Vancouver), vol. I, 1974, pp. 423-426.

8a. K. Kodaira, On compact analytic surfaces. II, Ann. of Math. (2) 77 (1963), 563-626.

8b. (1966), 682-721; ibid. 90 (1968), 55-83, 1048-1065.

8c. _ Pluricanonical systems on algebraic surfaces of general type, J. Math. Soc. Japan 20 (1968), $170-192$.

9. M. Lubke, Beweis einer Vermutung von Hartshorne für den Fall homogener Mannigfaltigkeiten, J. Reine Angew. Math. 316 (1980), 215-220.

10. Vo Van Tan, On the compactification of strongly pseudoconvex surfaces, Proc. Amer. Math. Soc. 82 (1981), 407-410.

Department of Mathematics, Suffolk University, Boston, Massachusetts 02114 